\title{
LA MISSIONE EVANGELIZZATRICE DELLA CHIESA NELLA COMUNITÀ PARROCCHIALE OGGI
}

\author{
Michele Cassese
}

Il mio contributo propone la riflessione vigente nella chiesa cattolica sulla missione evangelizzatrice della chiesa oggi, in particolare nella comunità parrocchiale. Tale riflessione viene accompagnata dall'analisi di esperienze concrete di evangelizzazione in alcune parrocchie italiane.

\section{La riscoperta del mandato missionario della chiesa}

Da alcuni anni nella chiesa cattolica romana si parla di "nuova evangelizzazione"; essa viene avvertita con una tale urgenza da spingere il papa Benedetto XVI a istituire nel 2010 Il Pontificio Consiglio per la Promozione della Nuova Evangelizzazione e indire il Sinodo dei vescovi del 2012 proprio su questo tema. Non si tratta di una questione specifica della chiesa cattolica né essa è circoscritta agli anni più recenti; è invece un problema centrale per le diverse chiese cristiane del secolo XX. Basta leggere la voce evangelizzazione curata da Emilio Castro, segretario generale del Consiglio Ecumenico delle Chiese (CEC), per il Dictionary of the Ecumenical Movement ${ }^{1}$, per verificare come il tema dell' evangelizzazione abbia coinvolto i dibattiti del CEC fin dal 1968 e i congressi degli Evangelicali dal 1966. Su di esso sono state

\footnotetext{
"Istituto di Studi Ecumenici "S. Bernardino" Venezia, Italia.

${ }^{1}$ Dizionario del Movimento Ecumenico, a cura di Nicolas Lossky, Jose Miguez Bonino, John Pobee, ed. it. a cura di G. Cereti, A. Filippi, L. Sartori, EDB, Bologna 1994, pp. 525-529.
} 
organizzate consultazioni all'interno del mondo dell'Ortodossia a partire dal 1974, e più in generale la dimensione dell'evangelizzazione è sentita in tutto il mondo della cristianità. Si tratta quindi di una questione comune.

Nella chiesa cattolica il richiamo alla missionarietà della chiesa e al suo compito di evangelizzazione è una riscoperta del Concilio Vaticano II (1962-1965). Si può dire con Luigi Sartori, che "la più grande svolta storica nella coscienza e nella vita della chiesa" operata dal Vaticano II è rappresentata dalla ripresa della dimensione missionaria della chiesa ${ }^{2}$. Alla missione della chiesa, infatti, il concilio ha dedicato un decreto specifico (Ad Gentes, AG), ma l'ha anche richiamata esplicitamente in altri documenti, in particolare nella costituzione dogmatica sulla chiesa (Lumen gentium, LG), nella costituzione pastorale su la Chiesa nel mondo contemporaneo (Gaudium et Spes, GS), nel decreto sull'apostolato dei laici (Apostolicam actuositatem, AA). La missione della chiesa ha rappresentato il filo rosso che attraversa tutti i documenti del Vaticano II.

Nei documenti conciliari la dimensione missionaria è vista in modo totalmente nuovo rispetto al passato: essa è essenza della chiesa, realtà che la qualifica nel profondo. "La chiesa peregrinante (sulla terra) per sua natura è missionaria" (AG 2): è missionaria, oppure non è più la chiesa di Gesù Cristo. Così inizia l'Ad Gentes: "Inviata per mandato divino alle genti per essere 'Sacramento universale di salvezza', la Chiesa, rispondendo alle esigenze più profonde della sua cattolicità e all'ordine specifico del suo Fondatore, si sforza di portare l'annuncio a tutti gli uomini" (AG 1).

La chiesa è istituita da Dio per la missione al mondo intero. "Sale della terra e luce del mondo", essa ha il compito di "salvare e rinnovare ogni creatura, perché tutte le cose siano ricapitolate in Cristo e gli uomini in Lui costituiscano una sola famiglia ed un solo

${ }^{2}$ L. Sartori, Dimensione missionaria della chiesa, in ID., Per una teologia in Italia, Scritti scelti, a cura di E. R. Tura, vol. I, Edizioni Messaggero, Padova 1997 , pp. 353-368, qui p. 356. 
popolo" (AG 1). La missione della chiesa ha origine dalla missione del Figlio (AG 3) e dello Spirito Santo inviato a continuare l'opera di salvezza di Cristo (AG 4). L'impegno di predicare la Buona Novella in tutto il mondo è assegnato direttamente da Cristo agli Apostoli e ai loro successori (AG 5). L'obiettivo è realizzare l'unità del genere umano e costituire delle diverse genti una sola famiglia, perché il disegno del Padre è che si formi un solo popolo di Dio. Questo impegno è allo stesso tempo un valore diffusivo di cui la Chiesa deve rispondere sul piano qualitativo dinanzi al suo Signore. Esso richiama l'apostolato in senso ampio, interessando tutti nella chiesa, sacerdoti e laici, celibi e sposati, uomini e donne: tutti sono chiamati alla partecipazione della missione della chiesa (AA 2).

Il concilio evidenzia anche che tale compito missionario va esercitato in sintonia e collaborazione innanzitutto con le altre chiese cristiane: pertanto la missionarietà della chiesa non consiste più nel convertire al cattolicesimo altri cristiani e uomini appartenenti ad altre credenze religiose, e non ha lo scopo di far nascere nuove chiese locali per una conversione massiccia al cattolicesimo, come si è creduto nel passato. Essa invece è una necessità della chiesa stessa per la salvezza degli uomini (LG 14), è un'impellenza dell' annuncio della verità salvifica (LG 17); "ricapitolare in Cristo" tutte le cose è contribuire alla realizzazione del disegno di Dio nella storia, far emergere la Sua cura per le sorti degli uomini, illuminare l'umanità sul Suo intervento salvifico nella vita degli uomini di tutti i tempi. Perciò questo va realizzato assieme alle altre chiese e comunità cristiane non cattoliche in uno spirito di collaborazione e non di antagonismo, di purificazione, scambio, aiuto e miglioramento reciproco; perciò la missione è una dimensione essenzialmente ecumenica. Il decreto sull'ecumenismo (Unitatis Redintegratio, UR) riconosce nelle altre chiese e comunità ecclesiali la presenza di "beni" di cui è munita e vivificata la stessa chiesa cattolica. Tali "beni" producono la vita della grazia e aprono alla salvezza (UR 3); anzi, delle chiese orientali (ortodosse) vien detto che hanno un "tesoro da cui ha attinto la Chiesa d'Occidente" e i dogmi fondamentali della fede cristiana (UR 14). Ancora la Chiesa cattolica 
riconosce "il contributo [che] hanno dato e danno cooperando insieme le altre chiese o comunità ecclesiali", per realizzare il medesimo compito (GS 40). Perciò nel cammino verso l'unità si richiamano i cristiani a una comune professione di fede davanti a tutti i popoli, a una mutua stima e testimonianza della speranza e cooperazione nel campo sociale (UR 12). Tale missione della chiesa infine non può eludere la collaborazione anche con le altre realtà religiose, soprattutto di ebrei e mussulmani, ma anche con quanti credono in un unico Dio, in uno spirito di fraternità e di pace (Nostra Aetate, NA 5).

\section{La "nuova evangelizzazione"}

Dopo il Concilio, i papi da Paolo VI a Francesco, come anche le Conferenze episcopali nazionali, hanno richiamato con insistenza tale compito specifico della chiesa, e lo presentano sempre più come la più urgente preoccupazione e principale cura dei pastori e della chiesa tutta. Fu Giovanni Paolo II a coniare nel 1979 l'espressione "nuova evangelizzazione" e la indicò come specifica missione della chiesa nel terzo millennio. Cosa si vuol intendere con l'espressione "nuova evangelizzazione"? Giovanni Paolo II puntualizzava che si tratta di presentare "il messaggio eterno della Rivelazione ....in un'ottica più conforme alla sensibilità della nostra epoca", di realizzare "un'evangelizzazione più attualizzata e più efficace", pertanto, ribadirà il suo successore Benedetto XVI, occorre "trovare adeguate forme per consentire ai nostri contemporanei di udire ancora la Parola viva ed eterna del Signore". E aggiungeva che è necessario "un attento discernimento" nella scelta di una forma appropriata nelle diverse situazioni, per cui la "nuova evangelizzazione" non significa trovare un' unica formula uguale per tutte le circostanze ${ }^{4}$. Essa deve essere concepita a partire dalle

${ }^{3}$ Giovanni Paolo II, Insieme per una nuova evangelizzazione. Lettera ai presidenti delle Conferenze episcopali d' Europa, 1986.

${ }^{4}$ Benedetto XVI, Ubicumque et semper, Motu proprio, 2010. 
situazioni sociali, culturali e religiose delle persone di un determinato territorio; è il contesto esistenziale e storico in cui si trovano gli interlocutori dell' annuncio cristiano a suggerire indirizzi pastorali più adatti e fecondi per l'evangelizzazione. Ancora più chiaramente il documento preparatorio del Sinodo del 2012 richiamava la necessità di "misurarsi con le sfide di un mondo in accelerata trasformazione" e trovare "la via per vivere oggi il dono dell' essere radunati dallo Spirito Santo a fare esperienza del Dio che ci è Padre, testimoniando e proclamando a tutti la Buona Notizia - il Vangelo - di Gesù Cristo" "Si tratta dunque di trovare forme più efficaci per collegare nella proposta del Vangelo due poli: l'eredità di fede ricevuta dai nostri Padri e le nuove situazioni e forme di vita. L'annuncio dell'Evangelo pertanto richiede ascolto e attenzione ai problemi e alle situazioni della nostra epoca. L'annuncio salvifico s'incarna nel mondo in cui noi stessi viviamo, e richiede un'attenta visione della società che ci circonda, una comprensione dei fenomeni da cui è coinvolta e risposte "evangeliche", che sanno cioè di "buona notizia", di Parola di Dio carica di speranza e di novità. Cos'ha da dire l'annuncio evangelico agli uomini del nostro tempo nei suoi diversi scenari? Si tratta di realtà drammatiche che si presentano agli occhi dell'osservatore attento. Sul piano culturale c'è il fenomeno di un distacco dalla fede, una visione secolarizzata della vita, mentre sul piano sociale si assiste a grandi trasformazioni mediante il fenomeno migratorio e il conseguente mescolamento di etnie e identità culturali, con ripercussioni sul legame ai valori tradizionali. Sul piano economico domina la globalizzazione accompagnata da una parte dalla crisi dei Paesi del vecchio Mondo, con la disoccupazione e fluttuazione del mercato del lavoro e del commercio, e dall'altra da un'emersione di alcuni Paesi in via di sviluppo, come Cina, Brasile e India con una rottura dei vecchi equilibri anche sul piano sociale, come un aumento del divario tra ceti alti e ceti bassi e la prevalenza

${ }^{5}$ Lineamenta 2011 (Sinodo dei Vescovi, XIII Assemblea Generale Ordinaria, La Nuova Evangelizzazione Per La Trasmissione Della Fede Cristiana. Lineamenta, 2011), Introduzione, n. 1. 
dell'economia finanziaria su quella reale. Preoccupante è d'altra parte il versante politico con attacchi all'ordine internazionale e agli Stati da parte delle forze terroristiche nate dal mondo islamico e afroasiatico, già prima della cosiddetta "primavera araba", come pure si verifica l'affermarsi di una gestione della politica affidata più alla leadership che alla democrazia. Infine, sul piano tecnico-scientifico avanza, pur tra tanti benefici sul piano della comunicazione, una cultura mediatica e digitale che sta modificando la comprensione della realtà e delle relazioni interpersonali. Nell'analisi su questa realtà fatta dalla gerarchia cattolica desta preoccupazione soprattutto la perdita del senso del sacro con tutto il patrimonio di verità cristiane e comprensione delle esperienze fondamentali della vita dell'uomo, come il nascere e il morire, la famiglia e le relazioni umane $^{6}$. In tutti questi scenari che suscitano angoscia, ansia e per alcuni disperazione, la chiesa cattolica era stata invitata dal Concilio Vaticano II a "conoscere e comprendere il mondo in cui viviamo", a cogliervi alla luce della fede "i segni dei tempi e di interpretarli alla luce del Vangelo" (GS 4), a "discerne negli avvenimenti, nelle richieste e nelle aspirazioni" degli uomini di questo tempo "quali sono i veri segni della presenza o del disegno di Dio" (GS 11). E oggi, dopo cinquant' anni, quale parola di speranza la chiesa cattolica può annunciare agli uomini del nostro tempo? Come rendere possibile e attuale quel messaggio di salvezza che è l'Evangelo? E in quale modo annunciarlo? Come far sì che la fede cristiana possa contribuire a guidare "l'intelligenza [umana] verso soluzioni pienamente umane" (GS 11)? Qui sta tutta la problematica relativa all'evangelizzazione oggi.

La risposta non può essere frutto d'improvvisazione né ripetizione di modalità esistenti, forse ormai inefficaci e poco feconde. Alle situazioni nuove insite in questi scenari deve

\footnotetext{
${ }^{6}$ Benedetto XVI, Ubicumque et semper, Motu proprio, 2010; cfr. anche Gilles Routhier, Un concilio per il XXI secolo. Il Vaticano II cinquant'anni dopo, Vita e Pensiero, Milano 2012, p. 45.
} 
corrispondere una nuova evangelizzazione nelle forme e negli atteggiamenti, ma forse anche negli attori.

Quello che mi pare certo è che metodo e stile dell' annuncio evangelico devono essere caratterizzati dalla stessa gioia annunciata dagli angeli ai pastori di Betlemme; caratteristica fortemente richiamata da papa Francesco nella sua esortazione apostolica Evangelii gaudium (EG) .

In tutte le situazioni occorre 1'“annuncio gioioso", uno "stile evangelizzatore" (EG 18) improntato alla gioia, perché la buona notizia dell'Evangelo è carica di gioia ed è ricca di speranza. "La gioia del Vangelo riempie il cuore e la vita di coloro che si incontrano con Gesù", perciò papa Francesco vuole invitare a percorrere "una nuova tappa evangelizzatrice marcata da questa gioia e indicare vie per il cammino della Chiesa nei prossimi anni" (EG 1).

In vista della nuova evangelizzazione, come richiama ancora il vescovo di Roma Francesco «la cosa di cui la chiesa ha più bisogno oggi è la capacità di curare le ferite e di riscaldare il cuore dei fedeli, la vicinanza, la prossimità. Io vedo la Chiesa come un ospedale da campo dopo una battaglia. Ė inutile chiedere a un ferito grave se ha il colesterolo e gli zuccheri alti! Si devono curare le sue ferite. Poi potremo parlare di tutto il resto. Curare le ferite, curare le ferite... E bisogna cominciare dal basso» ${ }^{7}$. L'evangelizzazione richiede vicinanza agli uomini reali, alle loro situazioni più drammatiche. Non può esserci senza la cura di uomini concreti, incarnati nella loro storia d'oggi. Evangelizzazione e promozione umana procedono insieme, anzi spesso la prima segue la seconda, nel senso che ha diritto di esserci dopo aver dimostrato prossimità e condivisione totale.

7 A. Spadaro, Intervista a papa Francesco, in "La civiltà cattolica", 3918, anno 164,19 settembre 2013, pp. 461-462. 


\section{Una pastorale per la nuova evangelizzazione}

Se la nuova evangelizzazione non è una campagna di proselitismo, essa non è nemmeno una ri-evangelizzazione, come se occorresse un nuovo messaggio di fede. Piuttosto è un rendere attuale proprio quel messaggio, consegnato da Cristo e trasmesso dagli apostoli, in una pratica evangelizzatrice nuova. Si tratta di una pastorale rinnovata, adatta alla nostra epoca, qualitativamente adeguata alle necessità degli uomini e delle donne del nostro tempo, attuata in forme che permettano di ascoltare e comprendere la Parola di salvezza. Si tratta dunque di dare un carattere contestuale e quindi "storico" all'annuncio evangelico. L'evangelizzazione nuova parte dalle sfide del mondo contemporaneo e risponde alle aspettative di questo stesso mondo.

L'altra condizione fondamentale per tale contestualità dell'azione evangelizzatrice, però, è quella del linguaggio per l'annuncio. Non può non essere lo stesso degli uomini a cui si rivolge, e perciò deve assumere le forme delle culture del tempo e del luogo; la Parola autenticamente evangelizzatrice è fatta di "parole di carne viva", è quella delle stesse donne e degli stessi uomini concreti a cui parla. Non si tratta solo dell'uso della lingua volgare, - aspetto ormai acquisito - ma di un linguaggio umano le cui modalità di espressione siano cariche di calore, mentre i contenuti emergono dall'esperienza personale e da una vita interiore profonda; un annuncio che usa parole che partono dal cuore di chi annuncia per arrivare al cuore e alla mente di chi ascolta. In definitiva un linguaggio empatico che comunichi speranza e miri alla trasformazione esistenziale. Solo la familiarità di un linguaggio "amico" rende attraente e penetrante il contenuto di quanto si annuncia.

Fondamentale, in questa prospettiva, è l'aspetto relativo agli attori della nuova evangelizzazione. Essa non può più essere un'azione di una sola parte della chiesa, il clero, ma è propria di tutta la comunità ecclesiale, di tutti i credenti. Per questo occorre superare le vecchie visioni ecclesiologiche che hanno guidato la chiesa 
cattolica fino alla metà del Novecento. Il Concilio Vaticano II, pur richiamando l'intenzione di Cristo, suo fondatore, di dare alla chiesa guide e ministeri, ha proposto con forte evidenza l'immagine di chiesa come popolo di Dio; un gregge unico che è pascolato dallo stesso pastore, Cristo. Lo dichiara con molta chiarezza papa Francesco: «Essere Chiesa significa essere Popolo di Dio, in accordo con il grande progetto d'amore del Padre. Questo implica essere il fermento di Dio in mezzo all'umanità. Vuol dire annunciare e portare la salvezza di Dio in questo nostro mondo, che spesso si perde, che ha bisogno di avere risposte che incoraggino, che diano speranza, che diano nuovo vigore nel cammino» (EG, 114).

Tutta la chiesa è missionaria, e tutti i credenti, clero e laici, religiosi e consacrati secolari, sono chiamati a esercitare tale compito missionario. Il documento conciliare sui laici è in ciò molto esplicito: essi partecipano alla missione della chiesa e all'evangelizzazione principalmente nei campi propri della famiglia, dei giovani, dell' ambiente sociale, dell' ordine nazionale e internazionale (AA 1114), ma prima di ogni altra cosa nella vita interna della comunità ecclesiale (AA 10). La funzione dei laici nell'opera evangelizzatrice della chiesa è dunque ineludibile, all'interno come all'esterno della comunità ecclesiale. Viene riconosciuto dalla Conferenza Episcopale Italiana (CEI) che "ogni battezzato ha ricevuto da Dio una personale chiamata alla edificazione e la crescita della comunità"; la chiesa è una unità comunionale di ricchezze personali, carismi da mettere a disposizione della "vita e della crescita del corpo ecclesiale e da far concorrere al riconoscimento della signoria di Cristo"; "dall' unità in Cristo scaturisce l'impegno a vivere questo dono nei diversi ambiti della vita", famiglia, vita sociale ${ }^{8}$. L'arcivescovo milanese Carlo Maria Martini commentando la seconda lettera ai Corinti sosteneva che "la cooperazione dei laici alla diffusione del Vangelo è fioritura della chiesa, è gioia del vescovo, è splendore del cielo, è pienezza di

${ }^{8}$ CEI, Educare alla vita buona del Vangelo, n.35; in ECEI, 8 (Enchiridion della Conferenza Episcopale Italiana, 8 (2006-2010), EDB, Bologna 2011) nn.3821-3822, p. 1825. 
vita cristiana". Quella cooperazione rappresenta "l'unico modo per cui la chiesa può diventare missionaria oggi con cui allargare le sue frontiere"s.

Per mettere in atto questa "nuova" visione di chiesa e una valorizzazione dei carismi e dei diversi ministeri al suo interno si richiede una "conversione" di tutta la comunità ecclesiale, del clero come dei laici. Tale conversione include cambiamento di mentalità e superamento di privilegi, purificazione e rinnovamento personale ma anche delle strutture delle comunità e della gestione dei ruoli in essa affidati. Una chiesa rinnovata è quella che libera e rivitalizza tutte le "energie del proprio corpo (...) per un annuncio e una proclamazione del Vangelo attraverso vie nuove, capaci di parlare alle culture odierne"10. Si tratta quindi di mettere in atto una "pastorale integrata", vale a dire "mettere in rete tutte le molteplici risorse, anche quelle umane, di ogni realtà e di ogni soggetto ecclesiali, in vista della comunicazione della fede" in un mondo in trasformazione ${ }^{11}$; è un incrociarsi dell'azione della chiesa e di tutti i suoi membri con quanto vivono gli uomini d'oggi; una pastorale che assuma modalità capaci di unire "fede amica dell'intelligenza e una prassi di vita caratterizzata dalla carità e dall'attenzione ai poveri e ai sofferenti"12. Per la comunità ecclesiale d'oggi questo significa recuperare sempre di più l'attenzione alle persone e alle famiglie, e non basta "attendere" le persone in chiesa, ma "andare" da loro e avvicinarle soprattutto nella vita concreta e quotidiana, entrare nelle case in cui abitano, nel loro vissuto quotidiano, nei luoghi in cui lavorano, usare i linguaggi che esse usano, comprendere l'atmosfera culturale che respirano, i problemi da cui sono oberati, le difficoltà

${ }^{9}$ C.M. Martini, Parole sulla Chiesa. Meditazioni sul Vaticano II, PIEMME, Casale Monferrato (AL) 1986, pp. 83-84.

${ }^{10}$ Lineamenta 2011, n. 5.

${ }^{11}$ S. Mazzolini, Il primato della missione evangelizzatrice della Chiesa. Una rilettura del IV Convegno ecclesiale nazionale (Verona, 16-20 2006), in " Euntes Docete", a. LX, 2 (2007), pp. 47- 65, qui p. 59.

${ }^{12}$ Benedetto XVI, L'Italia: bisognosa e favorevole, in "Il Regno-documenti", 19 (2006), p. 675. 
da affrontare, "annunciando e testimoniando, personalmente e comunitariamente, Cristo, speranza del mondo"13. "È questo il senso e il nocciolo di quella 'conversione pastorale' di cui sentiamo diffusa l'esigenza" 14 . Dunque un modello comunionale di chiesa come comunità di credenti in cui tutti si sentono corresponsabili della missione della chiesa, trovano possibilità di spazi riconosciuti di pastoralità ed operano nella condivisione di un comune obiettivo e nella collaborazione reciproca sia pure in ambiti di diversi.

\section{La parrocchia: luogo di evangelizzazione e di educazione alla fede}

Luogo privilegiato della missione della chiesa e quindi della "nuova evangelizzazione" è per la chiesa cattolica la parrocchia, la struttura più significativa nella vita del credente, che nella forma attuale risale a oltre sei-sette secoli fa e che ha ricevuto la sua definizione nel concilio di Trento (1545-1563). Da allora in poi essa è stata considerata in senso giuridico-canonico una chiesa con un territorio ben preciso, più o meno vasto, che cura il servizio spirituale agli abitanti di quella zona, vale a dire l'amministrazione dei sacramenti e il servizio divino ${ }^{15}$. Il popolo doveva pensare a sovvenire al sostentamento del parroco ${ }^{16}$, che per il concilio tridentino non è più il funzionario più o meno impegnato nel servizio divino senza rendere ragione del suo operato alla legge ecclesiastica, ma un pastore che si assume davanti a Dio e al suo vescovo la responsabilità delle anime a lui affidate e anche delle cose sacre -

${ }^{13}$ S.Mazzolini, Il primato della missione evangelizzatrice della Chiesa, p. 64 ${ }^{14}$ C.Ruini, La missione della Chiesa, la vita della società, I in "Il Regnodocumenti", 19 (2006), p. 691.

${ }^{15}$ COD (Conciliorum Oecumenicorum Decreta, a cura dell'Istituto per le scienze religiose, ediz. bilingue, EDB, Bologna 1991) 729, 36-41.

${ }^{16}$ COD, 730, 5-8. 
edificio, arredi e materiali - posti sotto la sua giurisdizione ${ }^{17}$. Talvolta, come nel Settecento, è anche un funzionario dello Stato che ratifica e concede attestati di cittadinanza e riscuote alcune imposte. Insomma era l'unico "punto di riferimento della vita parrocchiale" e come tale doveva conoscere i suoi parrocchiani e i loro problemi ${ }^{18}$. Ancora oggi la parrocchia è la chiesa ove vengono celebrati i momenti più importanti dell'esistenza. come l'accoglienza alla vita di fede col battesimo, il sacramento del matrimonio, e il commiato da questo mondo, con il funerale; in essa generalmente si ricevono i sacramenti dell'iniziazione cristiana, oltre il battesimo, l'eucaristia e la cresima, si ricevono i rudimenti della catechesi e, per i frequentanti, ci si incontra (settimanalmente) con il Cristo nella Parola e nell'Eucaristia.

Negli ultimi decenni sembra che la parrocchia stia perdendo terreno; è calata fortemente la partecipazione ai sacramenti, la vita si svolge in luoghi (scuola, centri commerciali, posto di lavoro, discoteca, pub, stadi ...) lontani dal campanile; per molti la vita stessa di fede avviene fuori della parrocchia o comunque non seguendo la comune divisione territoriale. Numerosi credenti cattolici hanno trovato maggiore risposta al loro bisogno spirituale ed esistenziale all'interno di movimenti religiosi laicali, per lo più riconosciuti ufficialmente dalle autorità romane.

Nonostante questo appannamento, papa Francesco non considera la parrocchia come una struttura caduca, tramontata, ma come un'istituzione, anche se non l'unica, dalla grande potenzialità evangelizzatrice; essa è "la chiesa stessa che vive in mezzo alle case dei suoi figli e delle sue figlie"19; "è comunità di comunità, santuario dove gli assetati vanno a bere per continuare a camminare, e centro

${ }^{17}$ G. G. Meersseman, Il tipo ideale di parroco secondo la riforma tridentina nelle sue fonti letterarie, in Il Concilio di Trento e la riforma tridentina, vol. I, Herder, Roma-Freiburg basel Baercellona Wien 1965, pp. 27- 44, qui p. 29.

${ }^{18}$ M. Guasco, Storia del clero in Italia dall'Ottocento a oggi, Laterza, RomaBari 1007, pp. 187, 228s.

${ }^{19}$ Il papa riprende la definizione di parrocchia del famoso documento pontificio Chrisifideles laici(1988), n. 26) 
di costante invio missionario". Egli invita tutte le altre istituzioni, associazioni e movimenti ecclesiali ad avere contatto con la realtà parrocchiale e "integrarsi nella pastorale organica della chiesa particolare", rappresentata dalla parrocchia (EG 28). I vescovi la ripropongono per il suo valore formativo, come "il primo luogo dell'educazione cristiana", "il punto ideale di riferimento e di convergenza" di tutta l'azione pastorale della chiesa diocesana ${ }^{20}$. Essi, rifacendosi alla bella immagine di papa Giovanni XXIII, vedono la parrocchia come la fontanella del villaggio a cui ogni abitante assetato va ad abbeverarsi; anche coloro che non la frequentano assiduamente, anzi anche quelli che ne sono lontani; essa ha acqua per tutti, in tutte le circostanze e in tutti i momenti della vita, nei momenti di gioia ma anche di tristezza e della prova ${ }^{21}$.

Una tale importanza porta così a considerare la parrocchia come un bisogno forte nel futuro della chiesa, come il luogo privilegiato in cui la comunità cristiana "genera la fede nel quotidiano della vita della gente" formazione della coscienza adulta; propone esperienze di libera e cordiale appartenenza, di servizio e di promozione sociale, di aggregazione e di festa" 23 . Essa è particolarmente "vicina al vissuto delle persone e agli ambienti di vita, rappresenta la comunità educante più completa in ordine alla fede. Mediante l'evangelizzazione e la catechesi, la liturgia e la preghiera, la vita di

20 C. M. Martini, Itinerari educativi. Seconda lettera per il programma pastorale 'Educare', Milano 1988, p. 114.

${ }^{21}$ Vedi la Lettera pastorale di Giuseppe Molinari, arcivescovo dell'Aquila, (2003): "La fontana del villaggio".

${ }^{22} \mathrm{R}$. Corti, La parrocchia (chiesa che vive) tra le case degli uomini, in "Il Regno-documenti”, 21/2003, pp. 665-674, qui p. 666.

23 Educare alla vita buona del Vangelo. Orientamenti pastorali dell'Episcoapato Italiano per il decennio 2010-2020, n. 39; in E/CEI, 8 [Enchiridion della Conferenza Episcopale Italiana Italiana, 8 (2006-2020), EDB, Bologna 2011], n. 3835; p. 1829. 
comunione nella carità, offre gli elementi essenziali del cammino del credente verso la pienezza della vita in Cristo" 24

Sia pure lentamente si avverte un cambiamento anche da parte del clero e dei fedeli circa la visione della pastorale parrocchiale, anche se ahimè si tratta di una realtà minoritaria in molte realtà ecclesiali italiane. Laddove vi è un parroco aggiornato e dinamico, coadiuvato da alcuni laici sensibili, la parrocchia tende a diventare, anche se con fatica, difficoltà e talvolta intoppi, una comunità di credenti che vivono una comunione di fede, di speranza e di carità in una coesione corale nell' ascolto della Parola e nella celebrazione del Signore nell'Eucaristia. La parrocchia allora è percepita come un insieme di con-vocati (chiamati assieme) dal Signore che accolgono il Suo richiamo a vivere nella dimensione comunitaria un impegno fattivo nella realtà ecclesiale e nel mondo. Il parroco in tal caso diventa il collante della comunità, colui che valorizza i carismi dei singoli e coordina i ministeri affidati ai diversi membri della parrocchia. La fontanina dunque è tutta la comunità che sotto la guida del parroco vive l'esperienza di fede nella comunione con i fratelli della parrocchia e allarga l'opera dei suoi credenti al di fuori delle mura dell' ambiente ecclesiale. È dunque tutta la comunità che si sente operatrice di pastoralità.

Questo nuovo atteggiamento è favorito anche dalle disposizioni date dal codice di diritto canonico che prevede per ogni parrocchia l'istituzione di un Consiglio pastorale parrocchiale e di un Consiglio per gli affari economici, entrambi eletti da un' assemblea o comunità parrocchiale. Il Consiglio pastorale parrocchiale dovrebbe essere rappresentativo delle varie realtà generazionali e dei diversi gruppi attivi nella comunità parrocchiale. All'atto pratico, purtroppo, spesso quest'organo è solo un luogo di presa visione delle disposizioni del parroco; oppure è luogo di formale approvazione di quanto già stabilito dal parroco stesso. Tuttavia non sono rare le esperienze di Consigli pastorali parrocchiali concepiti come momenti di formazione spirituale dei membri che vi partecipano, luogo di

${ }^{24}$ Educare alla vita buona del Vangelo, n. 39; in E/CEI, 8, n. 3836, p. 1829. 
dialogo e di approfondimento di problemi della parrocchia, e soprattutto spazio vitale per una progettazione della pastorale della comunità.

\section{Il Progetto pastorale parrocchiale}

Negli ultimi decenni emerge con forza la proposta della Conferenza Episcopale Italiana, e di alcuni vescovi in particolare, di approntare un progetto o piano pastorale da parte di ogni comunità parrocchiale. La proposta viene corredata di indicazioni teoriche ed operative. Tra le diverse indicazioni spiccano quelle date dall'arcivescovo Carlo M. Martini nel 1988, secondo il quale il progetto pastorale deve incarnarsi nel contesto sociale e religioso del territorio e guardare ad un tempo di almeno cinque-sei anni. È chiesto di individuare con chiarezza gli obiettivi e il senso del cammino, di definire con precisione le tappe da percorrere, di indicarle alla comunità in un programma annuale da realizzare con il "coinvolgimento di tutti i carismi e le forze operanti nell'ambito della parrocchia" 25 . Per analizzare questo tipo di percorsi ho preso in esame tre documenti di carattere orientativo della pastorale parrocchiale contemporanea: quelli dell'arcivescovo Martini; quello della famiglia salesiana affidataria di una parrocchia; e infine quello del centro pastorale Mondo Migliore, redatto in libretto da Nicola De Martini $^{26}$. Per studiarne la concreta realizzazione, ho esaminato tre progetti pastorali approntati in altrettante parrocchie del Nord e Sud Italia $^{27}$. Vale la pena di rilevare che questi tre progetti sono stati il

${ }^{25}$ C. M. Martini, Itinerari educativi, pp. 114- 115.

${ }^{26}$ Si tratta di C. M. Martini, Itinerari educativi, già citato e del progetto di Parrocchia affidata ai salesiani, in La Pastorale Giovanile Salesiana. Quaderno di riferimento, a cura del Dicastero per la Pastorale giovanile salesiana, Roma 2014; N. De Martini, Parrocchia nuova per tempi nuovi, LDC, Leumann (TO) 1985; (Il libretto è anche online dal Maggio 1997).

${ }^{27}$ Il primo è quello della Parrocchia di S. Maria del Rosario alla Starza, di Castellammare di Stabia (NA), realizzato a partire dagli inizi del 1980 e portato avanti fino al 1995 (di cui ho esperienza diretta per incontri con il parroco don 
frutto di un lavoro corale, cui ha partecipato un numero significativo di membri della parrocchia oppure specificamente della Consulta e del Consiglio pastorale almeno per un anno, con riunioni mensili e talvolta quindicinali nelle quali il progetto pastorale ha preso forma grazie alla discussione e alla condivisione attorno ad alcuni temi fondamentali. Tra questi, il modello di parrocchia da incarnare, gli obiettivi dell'azione pastorale e i suoi attori-ministri, il centro della Comunità, il senso e le modalità di celebrazione dell'eucaristia e della liturgia, la vita di carità in rapporto alla missionarietà della chiesa. Il progetto è stato di volta in volta stilato da un membro laico o laica, talvolta da un presbitero sulla base del materiale raccolto. In seguito il Consiglio parrocchiale e poi l'Assemblea l'hanno approvato $^{28}$. Le parrocchie infine hanno lavorato ogni anno alla stesura di un programma particolare focalizzato su un tema specifico del progetto pastorale. Tale programma era sempre soggetto a verifica alla fine dell' anno liturgico da parte dell' Assemblea.

Dall'analisi dei documenti si evince innanzitutto l'attenzione alla situazione storico-ambientale generale del nostro tempo e quella della realtà sociale, culturale e religiosa della singola parrocchia o territorio cittadino. La parrocchia è descritta nei progetti essenzialmente come luogo privilegiato di evangelizzazione, di educazione e di cammino di fede alla sequela del Signore ${ }^{29}$. Il modello operativo proposto è per tutti quello offerto negli Atti degli Apostoli $(2,42-44)$ : una comunità che vive nella gioia e nella carità l'esperienza dell'ascolto della parola degli Apostoli, l'unione

Vincenzo Scognamiglio e di partecipazione a momenti di attività parrocchiale); il secondo è quello della comunità parrocchiale S. Pio V di Milano, diretta allora dal parroco don Luigi Manganini, di cui si ha anche la pubblicazione a stampa del progetto (Parrocchia di S. Pio V e S. Maria di Calvairate Milano, Progetto Pastorale 1989-1994, Milano 1989); un terzo è quello della Parrocchia Ss. Pietro e Paolo di Santena (Torino ), Piano pastorale 2008-2014, pubblicato online.

${ }^{28}$ E' il caso della Parrocchia di S. Pio V e S. Maria in Calvairate di Milano; vedi il loro p. 3 .

${ }^{29}$ Ibidem, p. 116. 
fraterna, l'eucaristia e la preghiera. Tuttavia l'esponente di Mondo Migliore mette maggiormente in risalto il modello offerto da Marco (cf Mc 8,1-9), secondo il quale il comportamento pastorale è quello di Gesù che, nel deserto, moltiplica i pani e sfama la moltitudine. I1 popolo è affamato del pane della parola, della grazia, della fraternità: bisogna dar da mangiare alla moltitudine. Il primo modello pone il suo baricentro all'interno della realtà parrocchiale, che accoglie, alimenta spiritualmente i suoi membri, cresce e si nutre della relazione con Cristo e di quella tra i componenti della comunità, mira a dissetare gli uomini che vi accorrono e quelli del territorio del cui bisogno si ha notizia; l'altro propone l'istanza di uscire e andare incontro al "popolo", al mondo affamato e assetato. In questo caso, la comunità parrocchiale che pur vive della Parola e della grazia sacramentale si fa pellegrina e annunciatrice concreta al territorio della Parola di salvezza e del pane di grazia, testimoniando la condivisione.

Entrambi i modelli però mettono al centro l'annuncio della Parola di Dio, perché "la Chiesa non evangelizza se non si lascia evangelizzare". Si tratta di ascolto ma anche di celebrazione della Parola, soprattutto nell'Eucaristia, perché è essa che "alimenta e rafforza interiormente i cristiani e li rende capaci di un'autentica testimonianza evangelica nella vita quotidiana". (EG, 174). Per questo tra le parrocchie esaminate, quella di S. Pio V di Milano, seguendo proprio gli orientamenti del suo arcivescovo Martini, ha fatto della Lectio divina il centro della propria pastorale evangelizzatrice, con la creazione di gruppi di ascolto della Parola che si radunavano in parrocchia o nelle case delle famiglie. Il parroco a sua volta in un giorno preciso della settimana (martedì o mercoledi) teneva la lectio con incontri destinati a diverse fasce d'età o gruppi sociali. In quest'esperienza, la pista dell'anno era un libro o una lettera del Nuovo Testamento, letto in modo sistematico con tappe di proclamazione, meditazione, preghiera e condivisione di quanto annunciato ad ognuno dalla Parola, e con l'impegno di viverla anche fuori della mura degli edifici della parrocchia. Un'altra parrocchia, quella di Castellammare, ha creato ogni anno un nuovo gruppo di 
ascolto strutturato attorno a un testo, Shalom di Giuseppe Florio, che proponeva un percorso sulla chiamata di Dio rivolta all'uomo e al Suo popolo, Israele prima e la cristianità dopo, alla sua sequela e alla testimonianza nel mondo. In questo modo nell' arco di 15 anni sono sorte 15 piccole comunità di ascolto che hanno percorso per almeno 7 anni ciascuna un cammino di formazione. Dopo l'Esodo (al terzo anno) ciascun membro del gruppo d'ascolto era invitato a scegliere un impegno concreto nell'ambito della parrocchia - catechesi, liturgia e canto, solidarietà con le missioni lontane, il centro Caritas, i servizi quotidiani negli edifici della parrocchia, la Mensa domenicale parrocchiale per 150 immigrati, tra cui molti rumeni e ucraini, il settore di "Città viva" addetta allo studio e alla proposta di soluzione dei problemi socio-politici del territorio, o quello delle madri con bambini in difficoltà. Nelle parrocchie esaminate, pur privilegiando il cammino della Parola quale percorso formativo, si dà molto spessore anche alla catechesi a vari livelli: per i ragazzi che si preparano ai sacramenti d'iniziazione cristiana (Confessione, eucaristia e cresima), per i genitori che si preparano al battesimo dei loro bambini e affiancano $i$ figli nella loro preparazione ai sacramenti con un loro personale percorso catechetico, e per la comunità tutta almeno una volta al mese, su un tema scelto annualmente di carattere biblico-teologico. Si avverte una grande preoccupazione per la formazione dei formatori, quali primi corresponsabili del parroco della crescita della fede della parrocchia.

L'altro elemento che mi permetto di richiamare, come forse si è già colto, è proprio l'istanza di attenzione ai problemi della popolazione di tutto il territorio, perché la comunità parrocchiale 'piccola chiesa' si faccia segno di speranza anche per quanti non entrano in parrocchia. La carità di Dio di cui parla la Parola chiede di trovare concretezza in una risposta ai bisogni impellenti della comunità civile. Per questo alcune parrocchie organizzano centri di ascolto di casi bisognosi d'aiuto materiale e centri di ascolto per i problemi esistenziali e relazionali (Counseling spirituale). L'attività comprende anche, nella parrocchia milanese come in quella stabiese, collaborazioni con organizzazioni statali o comunali per la soluzione 
di casi meritevoli di particolare attenzione: uffici per la tossicodipendenza, pratiche burocratiche per anziani, malati e poveri, doposcuola per ragazzi e studenti in difficoltà $o$ anche per stranieri/extracomunitari/emigrati per l'insegnamento della lingua italiana. Si tratta di opere di supplenza o, come si dice da parte di alcuni, di sussidiarietà, in attesa che se ne facciano carico le istituzioni civili e politiche

Rimandando alla lettura dei documenti per un approfondimento maggiore della progettualità e programmazione della pastorale di alcune parrocchie-tipo nella chiesa cattolica in Italia, chiudo il mio contributo ricordando che come "ecclesia est semper reformanda", la chiesa deve sempre rinnovarsi, così la parrocchia se vuole essere veramente luogo privilegiato di nuova evangelizzazione in un mondo in continua trasformazione è chiamata a rivedere e rinnovare sempre la propria attività pastorale. Tale continuo aggiornamento sarà efficace e fecondo a tre condizioni: 1. la lettura della realtà storica considerata come "segno dei tempi", svelamento cioè di una necessità di far presente in una realtà concreta la redenzione del Cristo crocifisso e Risorto, mediante l'opera del Suo Spirito e l'azione "evangelizzatrice" della sua Chiesa; 2. il coinvolgimento di tutte le forze del popolo di Dio, dei credenti battezzati della sua comunità e il collegamento sinergico dei gruppi e delle piccole realtà presenti nella parrocchia; 3. l'aggancio concreto al territorio e la collaborazione con le realtà sociali, culturali, economiche e religiose, cristiane e non, in esso presenti. In tal modo la parrocchia diventa presenza e segno del Regno di Dio tra noi, di cui parlano i Vangeli e gli altri scritti neotestamentari. 